\title{
Primeira onda da Covid-19 em Juiz de Fora, MG, Brasil
}

\author{
Daniel M. Barbosa ${ }^{1}$ \\ IMECC/UNICAMP, Campinas, SP \\ Walter C. da S. Pires ${ }^{2}$ \\ DM/UFJF, Juiz de Fora, MG \\ Patrick de S. Oliveira ${ }^{3}$ \\ DM/UFMG, Belo Horizonte, MG \\ Lucy T. Takahashi ${ }^{4}$ \\ DM/UFJF, Juiz de Fora, MG
}

\begin{abstract}
Resumo. Neste estudo é apresentado um modelo epidemiológico compartimental, por meio do qual busca-se descrever a dinâmica da primeira onda da COVID-19 no município de Juiz de Fora, Minas Gerais, Brasil. A população é subdividida em nove compartimentos: suscetíveis, latentes, pré-sintomáticos, assintomáticos, sintomáticos com sintomas leves, sintomáticos com sintomas moderados, sintomáticos com sintomas severos, recuperados e mortos. Considera-se uma mudança de comportamento da população relacionada à ocupação dos leitos e das Unidades de Tratamento Intensivos hospitalares destinados aos pacientes com COVID-19 na cidade. Essa mudança é inserida na taxa de contágio dos indivíduos infecciosos que têm livre mobilidade: os pré-sintomáticos, os assintomáticos e os com sintomas leves. Nas simulações, os números totais de infectados e de mortos ficaram, ao longo do tempo, próximos aos números oficiais fornecidos pela Prefeitura de Juiz de Fora e pela Secretaria de Estado de Saúde de Minas Gerais.
\end{abstract}

Palavras-chave. SARS-CoV-2, Modelos Epidemiológicos, Juiz de Fora-MG, Dinâmica.

\section{Introdução}

A COVID-19 é uma doença respiratória aguda e altamente transmissível, que pode ocorrer em qualquer tipo de clima e é causada pelo coronavírus da síndrome respiratória aguda grave 2 (SARSCoV-2). É favorecida pelo maior número de contatos entre suscetíveis e infecciosos, como toda doença de transmissão direta. Esse coronavírus possui $70 \%$ de semelhança na sequência genética com o SARS-COV, vírus que causou a pandemia de H1N1 em 2009. A COVID-19 teve seu primeiro caso, no mundo, reportado em 31 de dezembro de 2019 em Wuhan, na República Popular da China, e no Brasil ocorreu em 26 de fevereiro de 2020, segundo o Ministério da Saúde [3] e em 11 de março a COVID-19 foi considerada pandêmica pela Organização Mundial da Saúde.

Neste trabalho, apresentamos um modelo matemático sobre a dinâmica da primeira onda da COVID-19 no município de Juiz de Fora, Minas Gerais, Brasil. Juiz de Fora está localizado na macrorregião Zona da Mata Mineira, vide 1, e trata-se de um importante polo industrial e comercial. Além disso, possui a maior população da macrorregião, estimada, pelo IBGE, em 573.285 pessoas em 2020, sendo $1 \%$ desta formada por profissionais da área da saúde que fazem testes de coronavírus periodicamente [5].

\footnotetext{
${ }^{1}$ danieldgbm68@gmail.com.

2 waltercesar0@gmail.com

${ }^{3}$ patrick-oliveira@ufmg.br

${ }^{4}$ ltiemi@gmail.com
} 


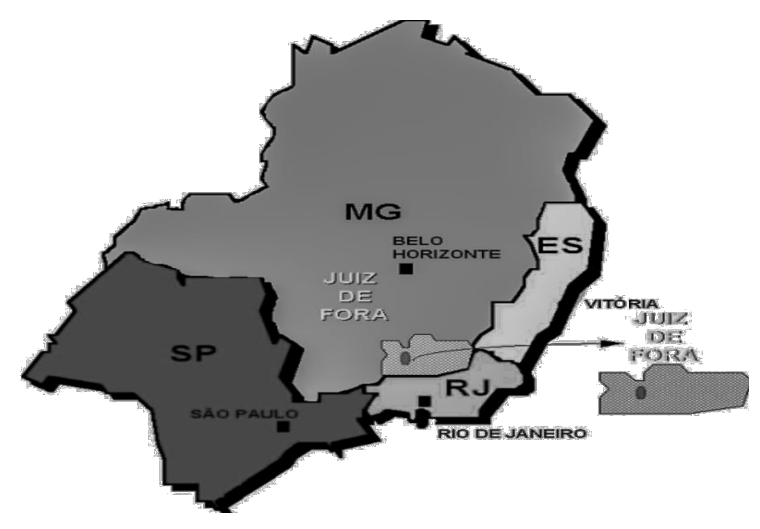

Figura 1: Mapa político da região sudeste do Brasil com destaque para Juiz de Fora na macrorregião Zona da Mata Mineira.

No município de Juiz de Fora, o Comitê Municipal de Enfrentamento à COVID-19, em 15 de maio de 2020, optou pelo isolamento social de acordo com as diretrizes do "Plano Minas Consciente: Retomando a economia do jeito certo" [2,7], criado pelo governo estadual. A retomada das atividades econômicas e da mobilidade da população depende da análise de alguns indicadores como, por exemplo, a porcentagem da ocupação das Unidades de Tratamento Intensivos (UTI's) Adulto destinadas especificamente aos pacientes com COVID-19 no município. Por meio da análise deste e de outros indicadores, a onda que a cidade está passando recebe uma das quatro cores: verde, branca, amarela ou vermelha. A ordem das cores está relacionada com a ordem de funcionamento de menor para maior nível de restrição, ditando, assim, quais estabelecimentos podem, ou não, voltar às atividades com os devidos cuidados sanitários e de biossegurança [2]. Dessa forma, supomos e agregamos ao modelo que, de acordo com a ocupação de leitos e UTI's destinados a COVID-19, a população responde com uma mudança de comportamento, fazendo com que a taxa de transmissividade do vírus se altere [6].

\subsection{Modelo Proposto}

Consideramos $N$ a população total, a qual está dividida em nove compartimentos, vide Tabela 1 .

Logo, para modelar a dinâmica da COVID-19, representamos as interações e relações entre os grupos descritos da seguinte maneira: os indivíduos Suscetíveis, $S$, após serem expostos ao SARSCoV-2 devido ao encontro com $I_{M}, I_{H}$ ou $\tilde{I},\left(\tilde{I}:=I_{a}+I_{s}+I_{L}\right)$, em períodos $\beta_{M}^{-1}, \beta_{H}^{-1}$ e $\beta(b)^{-1}$, respectivamente, podem se tornar Infectados latentes, $I$. Os indivíduos em $I$, após o período de incubação $\alpha^{-1}$, tornam-se Infecciosos Assintomáticos, $I_{a}$, em uma proporção $\varphi$, ou Infecciosos présintomáticos, $I_{s}$, numa proporção $(1-\varphi)$. Os indivíduos em $I_{s}$ migram, após um período $\nu^{-1}$, de acordo com os sintomas que venham a apresentar, para o compartimento dos Infecciosos com sintomas Leves, $I_{L}$, numa proporção $\phi_{1}$, ou para o de Infecciosos com sintomas Moderados, $I_{M}$, numa proporção $\phi_{2}$, ou, ainda, para o de Infecciosos com sintomas Severos, $I_{H}$, numa proporção $\left(1-\phi_{1}-\phi_{2}\right)$. Os indivíduos em $I_{a}$ e em $I_{L}$ não necessitam de cuidados especiais, se recuperam e migram para o compartimento dos Recuperados, $R$, após um período $\gamma_{1}^{-1}$. Já os indivíduos em $I_{M}$ e em $I_{H}$ necessitam de internações hospitalares em leitos e em UTI's, respectivamente. Após um período $\gamma_{2}^{-1}$, uma proporção $\sigma$ dos $I_{M}$ se recupera e migra para $R$, enquanto que os demais, $(1-\sigma) I_{M}$, vêm a óbito, sendo, então, transferidos para o compartimento de Mortos, $D$. Por fim, na subpopulação $I_{H}$, temos uma proporção $\eta$ que se recupera, enquanto os demais, $(1-\eta) I_{H}$, vêm a óbito, donde são transferidos para $R$ e $D$, respectivamente. Note que todos os 
Tabela 1: Descrição dos Compartimentos

\begin{tabular}{cc}
\hline Compartimento & Descrição \\
\hline Suscetível $(S)$ & $\begin{array}{c}\text { Indivíduos que não tiveram contato com } \\
\text { o vírus, ou seja, estão suscetíveis } \\
\text { a contrairem a doença. }\end{array}$ \\
\hline Infectado Latente $(I)$ & $\begin{array}{c}\text { Indivíduos que tiveram contato com } \\
\text { o vírus, porém não infectam ainda } \\
\text { devido a latência do vírus. }\end{array}$ \\
\hline Infeccioso Assintomático $\left(I_{a}\right)$ & Indivíduos que tiveram contato com \\
& vírus, porém não apresentaram sintomas. \\
\hline Infeccioso Pré-Sintomático $\left(I_{s}\right)$ & $\begin{array}{c}\text { Indivíduos que tiveram contato com vírus } \\
\text { e estão desenvolvendo os primeiros sintomas. }\end{array}$ \\
\hline Infeccioso com Sintomas Leves $\left(I_{L}\right)$ & $\begin{array}{c}\text { Indivíduos que tiveram contato com vírus } \\
\text { e apresentaram sintomas leves. }\end{array}$ \\
\hline Infeccioso com Sintomas Moderados $\left(I_{M}\right)$ & $\begin{array}{c}\text { Indivíduos que tiveram contato com vírus } \\
\text { e apresentaram sintomas moderados. }\end{array}$ \\
\hline Infeccioso com Sintomas Severos $\left(I_{H}\right)$ & $\begin{array}{c}\text { Indivíduos que tiveram contato com vírus } \\
\text { e apresentaram sintomas severos. }\end{array}$ \\
\hline Recuperados $(R)$ & Indivíduos que tiveram a doença e se \\
& recuperaram. \\
\hline Mortos $(D)$ & Indivíduos que morreram devido a doença. \\
\hline
\end{tabular}

parâmetros considerados são constantes e positivos, exceto a taxa $\beta(b)$, que depende diretamente do comportamento humano ao longo do tempo perante o cenário da COVID-19 no município. Assim, propomos o modelo (1) para a primeira onda da COVID-19 no município de Juiz de Fora.

$$
\left\{\begin{aligned}
\frac{d S}{d t} & =-\beta(b)\left(I_{a}+I_{s}+I_{L}\right) \frac{S}{N}-\beta_{M} I_{M} \frac{S}{N}-\beta_{H} I_{H} \frac{S}{N} \\
\frac{d I}{d t} & =\beta(b)\left(I_{a}+I_{s}+I_{L}\right) \frac{S}{N}+\beta_{M} I_{M} \frac{S}{N}+\beta_{H} I_{H} \frac{S}{N}-\alpha I \\
\frac{d I_{a}}{d t} & =\alpha I \varphi-\gamma_{1} I_{a} \\
\frac{d I_{s}}{d t} & =\alpha I(1-\varphi)-\nu I_{s} \\
\frac{d I_{L}}{d t} & =\nu I_{s} \phi_{1}-\gamma_{1} I_{L} \\
\frac{d I_{M}}{d t} & =\nu I_{s} \phi_{2}-\gamma_{2} I_{M}, \\
\frac{d I_{H}}{d t} & =\nu I_{s}\left(1-\phi_{1}-\phi_{2}\right)-\gamma_{2} I_{H} \\
\frac{d R}{d t} & =\gamma_{1} I_{a}+\gamma_{1} I_{L}+\gamma_{2} I_{M} \sigma+\gamma_{2} I_{H} \eta \\
\frac{d D}{d t} & =\gamma_{2} I_{M}(1-\sigma)+\gamma_{2} I_{H}(1-\eta) .
\end{aligned}\right.
$$




\section{Simulações e Resultados}

Utilizamos o software livre GNU Octave para realizar simulações do modelo (1), fazendo uso do método implementado a partir dos modelos Runge-Kutta de quarta ordem (ode45). Nessas simulações, para o município de Juiz de Fora, tomamos a população total $N=573.285$ e um período de 260 dias. Esse período abrange o início do número de novos casos reportados continuamente de COVID-19 [4] até o aparente surgimento da segunda onda, ou seja, do dia 27 de março ao dia 03 de dezembro de 2020. Para os parâmetros do modelo (1), os valores considerados são os dispostos na Tabela 2, que estão de acordo com [8], e as condições iniciais $S=99,995 \%, I_{H}=0,005 \%$ e as demais subpopulações nulas. Já a taxa $\beta$ de transmissão dos $\tilde{I}$ é uma função escada que depende da fração b, dada por $b=\frac{I_{H}}{195}$, sendo 195 corresponde ao total de leitos e UTI's disponibilizados em Juiz de Fora para os pacientes com COVID-19 [7]. Os quatro valores iniciais das faixas de $\beta(b)$ referem-se, respectivamente, a cada uma das quatro ondas dentro do programa Minas Consciente e são inversamente proporcionais aos valores assumidos por $b$, sendo escolhidos empiricamente de modo a descreverem o comportamento dos dados. Além disso, temos $\beta(b)=0$ correspondendo à ocupação total dos leitos e UTI's, vide Tabela 3.

Tabela 2: Valores dos parâmetros do modelo (1), segundo [8].

\begin{tabular}{cccccccccc}
\hline$\beta_{H}^{-1}$ & $\beta_{M}^{-1}$ & $\alpha^{-1}$ & $\varphi$ & $\nu^{-1}$ & $\phi_{1}$ & $\phi_{2}$ & $\gamma_{1}^{-1}$ & $\gamma_{2}^{-1}$ & $\eta$ \\
\hline 10 & 5 & 5 & 0,3 & 3 & 0,8 & 0,15 & 20 & 21 & 0,5 \\
\hline
\end{tabular}

Tabela 3: Taxa de transmissão $\beta$ em função da fração de leitos ocupados $b$.

\begin{tabular}{cccccc}
\hline & $b \leq 0,2$ & $0,2<b \leq 0,3$ & $0,3<b \leq 0,85$ & $0,85<b \leq 0,99$ & $b>0,99$ \\
\hline$\beta(b)$ & 0,1875 & 0,0625 & 0,0562 & 0,0468 & 0 \\
\hline
\end{tabular}

Para a validação dos dados simulados consideramos que o número total de infectados, $I_{T}$, seja dado por todos os indivíduos que foram testados positivamente para o SARS-CoV-2 em algum momento nesses 260 dias. Assim, tomamos

$$
I_{T}=I_{M}+I_{H}+\gamma_{2} I_{M} \sigma+\gamma_{2} I_{H} \eta+D+T
$$

sendo $T$ dado por

$$
T=0,01\left(I_{a}+I_{s}+I_{L}+\gamma_{1} I_{a}+\gamma_{1} I_{L}\right)
$$

que representa os profissionais da saúde, entre outros.

Por meio das simulações, construímos $I_{T}$, dado por (2), cujo resultado é exposto na Figura 2, onde a curva simulada está compatível com os dados fornecidos pela Secretaria de Estado de Saúde de Minas Gerais (SES) e pela PJF durante, os 260 dias de simulação [1,7]. Temos, também, compatibilidade com os dados da mortalidade no mesmo período, com exceção do período em torno do terceiro mês, vide Figura 3. Mas, após o $252^{\circ}$ dia, as curvas com dados empíricos $[1,7]$ do número de infectados e de mortos se distanciam da curva simulada, visto que têm maior crescimento. Portanto, esse período é o possível início da segunda onda de COVID-19 no município. 


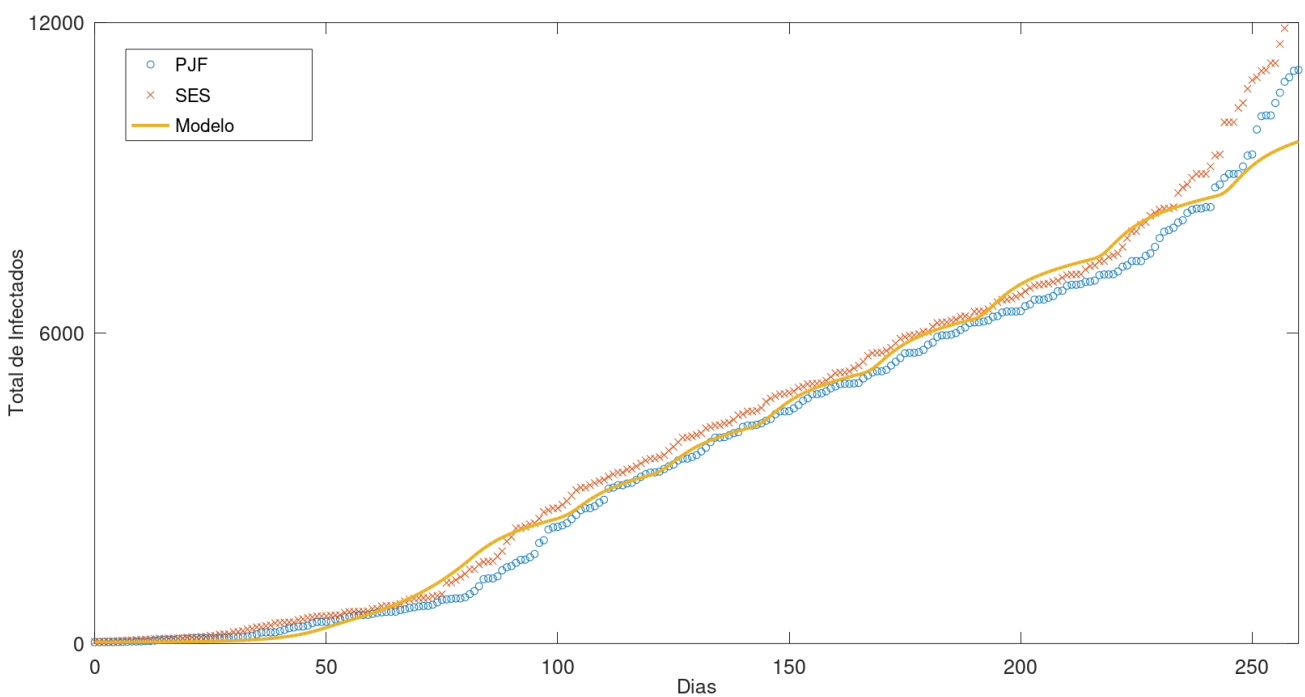

Figura 2: Total de infectados por COVID-19 disponibilizados pela PJF, em azul, e pela SES, em vermelho, e os simulados, em laranja, do dia 27 de março a 12 de dezembro de 2020, totalizando 260 dias de simulação. Fonte: Elaborado pelos autores.

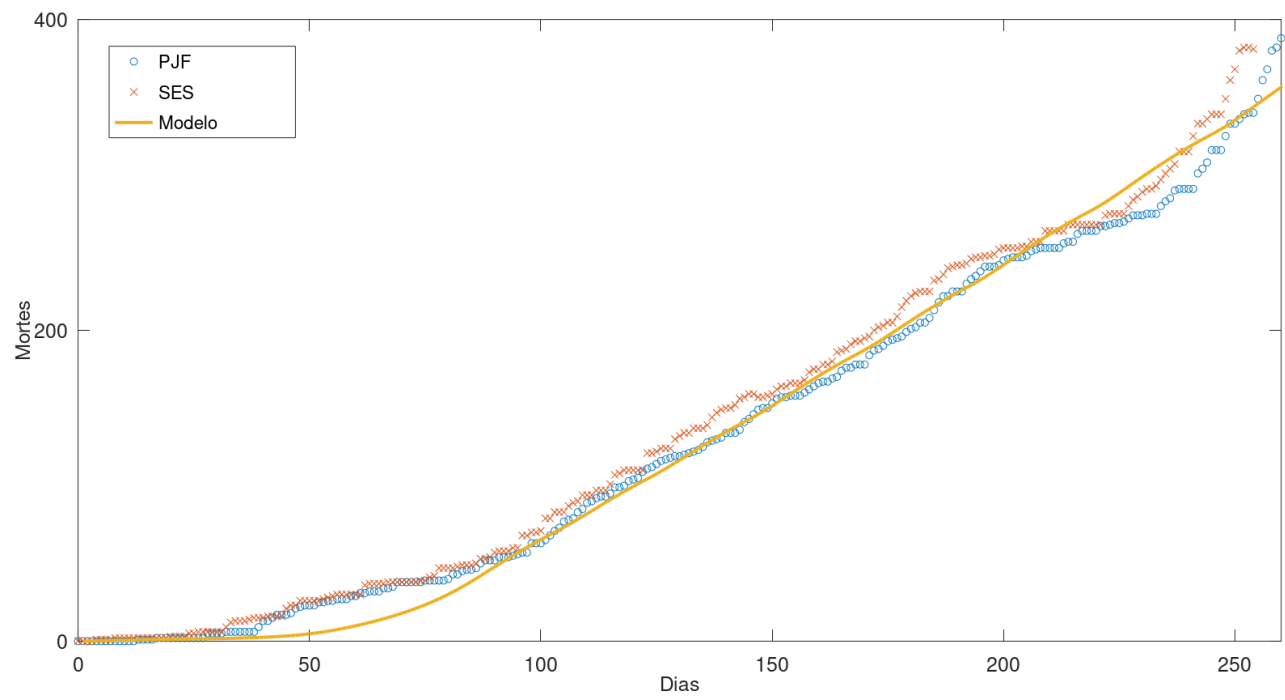

Figura 3: Total de mortes por COVID-19 disponibilizados pela PJF, em azul, e pela SES, em vermelho, e os simulados, em laranja, do dia 27 de março a 12 de dezembro de 2020, totalizando 260 dias de simulação. Fonte: Elaborado pelos autores.

\section{Conclusões}

Ao compararmos os dados empíricos com os simulados vemos que o modelo proposto descreve de forma satisfatória a primeira onda da COVID-19 em Juiz de Fora, que abrange o período de 27 
de março a 03 de dezembro de 2020, com um total de 251 dias. Entretanto, é perceptível que, após o $251^{\circ}$ dia, os dados da simulação do modelo deixam de ser compatíveis com os dados empíricos, o que pode sinalizar o início da segunda onda da COVID-19 no município. Logo, inferimos que as taxas de transmissão da doença aumentaram alguns dias antes do $251^{\circ}$ dia da simulação.

Concluímos, portanto, por meio das simulações, que os valores das taxas de transmissão dos infecciosos $\tilde{I}$ têm grande impacto no número total de casos, ou seja, esses indivíduos têm uma forte influência na dinâmica da COVID-19, segundo o modelo proposto. Para cada valor do parâmetro $\beta(b)$, vide Tabela 3 , temos uma solução do tipo exponencial que representa a dinâmica de acordo com a ocupação dos leitos e UTI's destinados aos pacientes com COVID-19 no município de Juiz de Fora.

\section{Agradecimentos}

O presente trabalho foi realizado com apoio parcial da Coordenação de Aperfeiçoamento de Pessoal de Nível Superior - Brasil (CAPES) - Código de Financiamento 001 e da Pró-Reitoria de Pós-Graduação e Pesquisa da UFJF - BIC/UFJF.

\section{Referências}

[1] Brasil, Ministério da Saúde, Secretaria de Estado de Saúde de Minas Gerais. Painel de Monitoramento de Casos. Disponível em: http://coronavirus.saude.mg.gov.br/. Acesso em: 30 de out. de 2020 .

[2] Brasil, Ministério da Saúde, Secretaria de Estado de Saúde de Minas Gerais.Plano Minas Consciente: Retomando a economia do jeito certo. Disponivel em: https://www.mg.gov.br/sites/default/files/paginas/imagens/minasconsciente/plano_minas_ consciente_3.3_v.5.pdf. Acesso em 15 de fev. de 2021.

[3] Brasil, Ministério da Saúde, Conselho Nacional de Saúde, Brasil confirma primeiro caso do novo coronavírus porém não há motivo para pânico. Disponível em: http://conselho.saude.gov.br/ultimas-noticias-cns/1042-brasil-confirma-primeiro-casodo-novo-coronavirus-porem-nao-ha-motivo-para-panico. Acesso em: 15 de fev. de 2021.

[4] Brasil, Ministério da Saúde, Secretaria de Estado de Saúde de Minas Gerais. BOLETIM INFORMATIVO DO COES MINAS COVID19. Disponivel em: https://coronavirus.saude.mg.gov.br/images/boletim/03marco/14032020_Boletim_epidemiologico_COVID-19_MG.pdf. Acesso em 15 de fev. de 2021.

[5] IBGE - INSTITUTO BRASILEIRO DE GEOGRAFIA E ESTATÍSTICA. Panorama da população de Juiz de Fora. Disponível em: https://cidades.ibge.gov.br/brasil/mg/juiz-defora/panorama. Acesso em 15 de fev. de 2021.

[6] Patrão, M. e Reis, M., Analisando a pandemia de COVID-19 através dos modelos SIR e SECIAR, Biomatemática, 30:111-140, 2020.

[7] PREFEITURA DE JUIZ DE FORA. Painel gerencial - principais dados covid, 2020. Disponível em: https://covid19.pjf.mg.gov.br/. Acesso em: 30 de out. de 2020.

[8] Lima, M., Leão, J., Cabral, C., Dias, S., et al. Relatório Técnico: Resposta ao Ofício n.0174/2020 - GP/FAPEAM - Curva de Contaminação Covid-19 Estado do Amazonas, Tech. Rep., 2020. 